\title{
Manifolds with a Lower Ricci Curvature Bound
}

\author{
Guofang Wei
}

\begin{abstract}
This paper is a survey on the structure of manifolds with a lower Ricci curvature bound.
\end{abstract}

\section{Introduction}

The purpose of this paper is to give a survey on the structure of manifolds with a lower Ricci curvature bound. A Ricci curvature bound is weaker than a sectional curvature bound but stronger than a scalar curvature bound. Ricci curvature is also special in that it occurs in the Einstein equation and in the Ricci flow. The study of manifolds with lower Ricci curvature bound has experienced tremendous progress in the past fifteen years. Our focus in this article is strictly restricted to results with only Ricci curvature bound, and no result with sectional curvature bound is presented unless for straight comparison. The reader is referred to John Lott's article in this volume for the recent important development concerning Ricci curvature for metric measure spaces by Lott-Villani and Sturm. We start by introducing the basic tools for studying manifolds with lower Ricci curvature bound (Sections 2-4), then discuss the structures of these manifolds (Sections 5-9), with examples in Section 10.

The most basic tool in studying manifolds with Ricci curvature bound is the Bochner formula. From there one can derive powerful comparison tools like the mean curvature comparison, the Laplacian comparison, and the relative volume comparison. For the Laplacian comparison (Section 3) we discuss the global version in three weak senses (barrier, distribution, viscosity) and clarify their relationships (I am very grateful to my colleague Mike Crandall for many helpful discussions and references on this issue). A generalization of the volume comparison theorem to an integral Ricci curvature bound is also presented (Section 4). Important tools such as Cheng-Yau's gradient estimate and Cheeger-Colding's segment inequality are presented in Sections 2 and 4 respectively. Cheeger-Gromoll's splitting

The author was partially supported by NSF grant DMS-0505733. 
theorem and Abresch-Gromoll's excess estimate are presented in Sections 5 and 8 respectively.

From comparison theorems, various quantities like the volume, the diameter, the first Betti number, and the first eigenvalue are bounded by the corresponding quantity of the model. When equality occurs one has the rigid case. In Section 5 we discuss many rigidity and stability results for nonnegative and positive Ricci curvature. The Ricci curvature lower bound gives very good control on the fundamental group and the first Betti number of the manifold; this is covered in Section 6 (see also the very recent survey article by Shen-Sormani [97] for more elaborate discussion). In Sections 7, 8, and 9 we discuss rigidity and stability for manifolds with lower Ricci curvature bound under Gromov-Hausdorff convergence, almost rigidity results, and the structure of the limit spaces, mostly due to Cheeger and Colding. Examples of manifolds with positive Ricci curvature are presented in Section 10.

Many of the results in this article are covered in the very nice survey articles $[\mathbf{1 1 8}, \mathbf{2 3}]$, where complete proofs are presented. We benefit greatly from these two articles. Some materials here are adapted directly from [23] and we are very grateful to Jeff Cheeger for his permission. We also benefit from $[\mathbf{4 9}, \mathbf{2 4}]$ and the lecture notes $[\mathbf{1 0 8}]$ of a topics course I taught at UCSB. I would also like to thank Jeff Cheeger, Xianzhe Dai, Karsten Grove, Peter Petersen, Christina Sormani, and William Wylie for reading earlier versions of this article and for their helpful suggestions.

\section{Bochner's formula and the mean curvature comparison}

For a smooth function $f$ on a complete Riemannian manifold $\left(M^{n}, g\right)$, the gradient of $\mathrm{f}$ is the vector field $\nabla f$ such that $\langle\nabla f, X\rangle=X(f)$ for all vector fields $X$ on $M$. The Hessian of $f$ is the symmetric bilinear form

$$
\operatorname{Hess}(f)(X, Y)=X Y(f)-\nabla_{X} Y(f)=\left\langle\nabla_{X} \nabla f, Y\right\rangle
$$

and the Laplacian is the trace $\Delta f=\operatorname{tr}(\operatorname{Hess} f)$. For a bilinear form $A$, we denote $|A|^{2}=\operatorname{tr}\left(A A^{t}\right)$. The most basic tool in studying manifolds with Ricci curvature bound is the Bochner formula. Here we state the formula for functions.

Theorem 2.1 (Bochner's Formula). For a smooth function $f$ on a complete Riemannian manifold $\left(M^{n}, g\right)$,

$$
\frac{1}{2} \Delta|\nabla f|^{2}=|\operatorname{Hess} f|^{2}+\langle\nabla f, \nabla(\Delta f)\rangle+\operatorname{Ric}(\nabla f, \nabla f) .
$$

This formula has many applications. In particular, we can apply it to the distance function, harmonic functions, and the eigenfunctions among others. The formula has a more general version (Weitzenböck type) for vector fields (1-forms), which also works nicely on Riemannian manifolds with a smooth 
measure $[\mathbf{7 0}, \mathbf{8 5}]$ where Ricci and all adjoint operators are defined with respect to the measure.

Let $r(x)=d(p, x)$ be the distance function from $p \in M . r(x)$ is a Lipschitz function and is smooth on $M \backslash\left\{p, C_{p}\right\}$, where $C_{p}$ is the cut locus of $p$. At smooth points of $r$,

$$
|\nabla r| \equiv 1, \quad \operatorname{Hess} r=I I, \Delta r=m,
$$

where $I I$ and $m$ are the second fundamental form and mean curvature of the geodesics sphere $\partial B(p, r)$.

Putting $f(x)=r(x)$ in $(2.1)$, we obtain the Riccati equation along a radial geodesic,

$$
0=|I I|^{2}+m^{\prime}+\operatorname{Ric}(\nabla r, \nabla r) .
$$

By the Schwarz inequality,

$$
|I I|^{2} \geq \frac{m^{2}}{n-1} .
$$

Thus, if $\operatorname{Ric}_{M^{n}} \geq(n-1) H$, we have the Riccati inequality,

$$
m^{\prime} \leq-\frac{m^{2}}{n-1}-(n-1) H .
$$

Let $M_{H}^{n}$ denote the complete simply connected space of constant curvature $H$ and $m_{H}$ the mean curvature of its geodesics sphere; then

$$
m_{H}^{\prime}=-\frac{m_{H}^{2}}{n-1}-(n-1) H .
$$

Since $\lim _{r \rightarrow 0}\left(m-m_{H}\right)=0$, using $(2.4),(2.5)$ and the standard Riccati equation comparison, we have

Theorem 2.2 (Mean Curvature Comparison). If $\operatorname{Ric}_{M^{n}} \geq(n-1) H$, then along any minimal geodesic segment from $p$,

$$
m(r) \leq m_{H}(r) .
$$

Moreover, equality holds if and only if all radial sectional curvatures are equal to $H$.

By applying the Bochner formula to $f=\log u$ with an appropriate cutoff function and looking at the maximum point one has Cheng-Yau's gradient estimate for harmonic functions $[\mathbf{3 4}]$.

Theorem 2.3 (Gradient Estimate, Cheng-Yau 1975). Let Ric $_{M^{n}} \geq$ $(n-1) H$ on $B\left(p, R_{2}\right)$ and $u: B\left(p, R_{2}\right) \rightarrow \mathbb{R}$ satisfying $u>0, \Delta u=0$. Then for $R_{1}<R_{2}$, on $B\left(p, R_{1}\right)$,

$$
\frac{|\nabla u|^{2}}{u^{2}} \leq c\left(n, H, R_{1}, R_{2}\right)
$$


If $\Delta u=K(u)$, the same proof extends and one has $[\mathbf{2 3}]$

$$
\frac{|\nabla u|^{2}}{u^{2}} \leq \max \left\{2 u^{-1} K(u), c\left(n, H, R_{1}, R_{2}\right)+2 u^{-1} K(u)-2 K^{\prime}(u)\right\} .
$$

\section{Laplacian comparison}

Recall that $m=\Delta r$. From (2.6), we get the local Laplacian comparison for distance functions

$$
\Delta r \leq \Delta_{H} r, \text { for all } x \in M \backslash\left\{p, C_{p}\right\} .
$$

Note that if $x \in C_{p}$, then either $x$ is a (first) conjugate point of $p$ or there are two distinct minimal geodesics connecting $p$ and $x[\mathbf{2 9}]$, so $x \in\{$ conjugate locus of $p\} \cup\{$ the set where $r$ is not differentiable $\}$. The conjugate locus of $\mathrm{p}$ consists of the critical values of $\exp _{p}$. Since $\exp _{p}$ is smooth, by Sard's theorem, the conjugate locus has measure zero. The set where $r$ is not differentiable has measure zero since $r$ is Lipschitz. Therefore the cut locus $C_{p}$ has measure zero. One can show $C_{p}$ has measure zero more directly by observing that the region inside the cut locus is star-shaped [18, Page 112]. The above argument has the advantage that it can be extended easily to show that Perelman's $l$-cut locus $[\mathbf{8 5}]$ has measure zero since the $\mathcal{L}$-exponential map is smooth and the $l$-distance function is locally Lipschitz.

In fact the Laplacian comparison (3.1) holds globally in various weak senses. First we review the definitions (for simplicity we only do so for the Laplacian) and study the relationship between these different weak senses.

For a continuous function $f$ on $M, q \in M$, a function $f_{q}$ defined in a neighborhood $U$ of $q$ is an upper barrier of $f$ at $q$ if $f_{q}$ is $C^{2}(U)$ and

$$
f_{q}(q)=f(q), \quad f_{q}(x) \geq f(x)(x \in U) .
$$

Definition 3.1. For a continuous function $f$ on $M$, we say $\Delta f(q) \leq c$ in the barrier sense ( $f$ is a barrier subsolution to the equation $\Delta f=c$ at $q$ ), if for all $\epsilon>0$, there exists an upper barrier $f_{q, \epsilon}$ such that $\Delta f_{q, \epsilon}(q) \leq c+\epsilon$.

This notion was defined by Calabi [17] back in 1958 (he used the terminology "weak sense" rather than "barrier sense"). A weaker version is in the sense of viscosity, introduced by Crandall and Lions in [38].

Definition 3.2. For a continuous function $f$ on $M$, we say $\Delta f(q) \leq c$ in the viscosity sense ( $f$ is a viscosity subsolution of $\Delta f=c$ at $q$ ), if $\Delta \phi(q) \leq c$ whenever $\phi \in C^{2}(U)$ and $(f-\phi)(q)=\inf _{U}(f-\phi)$, where $U$ is a neighborhood of $q$.

Clearly barrier subsolutions are viscosity subsolutions.

Another very useful notion is subsolution in the sense of distributions. 
Definition 3.3. For continuous functions $f, h$ on an open domain $\Omega \subset M$, we say $\Delta f \leq h$ in the distribution sense ( $f$ is a distribution subsolution of $\Delta f=h$ ) on $\Omega$, if $\int_{\Omega} f \Delta \phi \leq \int_{\Omega} h \phi$ for all $\phi \geq 0$ in $C_{0}^{\infty}(\Omega)$.

By [58] if $f$ is a viscosity subsolution of $\Delta f=h$ on $\Omega$, then it is also a distribution subsolution and vice versa, see also [66], [57, Theorem 3.2.11].

For geometric applications, the barrier and distribution sense are very useful and the barrier sense is often easy to check. Viscosity gives a bridge between them. As observed by Calabi [17] one can easily construct upper barriers for the distance function.

LEMMA 3.4. If $\gamma$ is minimal from $p$ to $q$, then for all $\epsilon>0$, the function $r_{q, \epsilon}(x)=\epsilon+d(x, \gamma(\epsilon))$ is an upper barrier for the distance function $r(x)=$ $d(p, x)$ at $q$.

Since $r_{q, \epsilon}$ trivially satisfies (3.2) the lemma follows by observing that it is smooth in a neighborhood of $q$.

Upper barriers for Perelman's l-distance function can be constructed very similarly.

Therefore the Laplacian comparison (3.1) holds globally in all the weak senses above. Cheeger-Gromoll (unaware of Calabi's work at the time) had proved the Laplacian comparison in the distribution sense directly by observing the very useful fact that near the cut locus $\nabla r$ points towards the cut locus [30], see also [23]. (However it is not clear if this fact holds for Perelman's $l$-distance function.)

One reason why these weak subsolutions are so useful is that they still satisfy the following classical Hopf strong maximum principle, see [17], also e.g., [23] for the barrier sense, see $[\mathbf{6 7}, \mathbf{6 0}]$ for the distribution and viscosity senses, also [57, Theorem 3.2.11] in the Euclidean case.

Theorem 3.5 (Strong Maximum Principle). If on a connected open set, $\Omega \subset M^{n}$, the function $f$ has an interior minimum and $\Delta f \leq 0$ in any of the weak senses above, then $f$ is constant on $\Omega$.

These weak solutions also enjoy regularity (e.g., if $f$ is a weak sub and sup solution of $\Delta f=0$, then $f$ is smooth), see e.g., [47].

The Laplacian comparison also works for radial functions (functions composed with the distance function). In geodesic polar coordinates, we have

$$
\Delta f=\tilde{\Delta} f+m(r, \theta) \frac{\partial}{\partial r} f+\frac{\partial^{2} f}{\partial r^{2}}
$$

where $\tilde{\Delta}$ is the induced Laplacian on the sphere and $m(r, \theta)$ is the mean curvature of the geodesic sphere in the inner normal direction. Therefore 
TheOREM 3.6 (Global Laplacian Comparison). If $\operatorname{Ric}_{M^{n}} \geq(n-1) H$, in all the weak senses above, we have

$$
\begin{array}{ll}
\Delta f(r) \leq \Delta_{H} f(r) & \left(\text { if } f^{\prime} \geq 0\right), \\
\Delta f(r) \geq \Delta_{H} f(r) & \left(\text { if } f^{\prime} \leq 0\right) .
\end{array}
$$

\section{Volume comparison}

For $p \in M^{n}$, use exponential polar coordinates around $p$ and write the volume element $d$ vol $=\mathcal{A}(r, \theta) d r \wedge d \theta_{n-1}$, where $d \theta_{n-1}$ is the standard volume element on the unit sphere $S^{n-1}(1)$. By the first variation of the area (see $[\mathbf{1 1 8}]$ )

$$
\frac{\mathcal{A}^{\prime}}{\mathcal{A}}(r, \theta)=m(r, \theta)
$$

Similarly, define $\mathcal{A}_{H}$ for the model space $M_{H}^{n}$. The mean curvature comparison and (4.1) gives the volume element comparison. Namely if $M^{n}$ has $\operatorname{Ric}_{M} \geq(n-1) H$, then

$$
\begin{aligned}
& \frac{\mathcal{A}(r, \theta)}{\mathcal{A}_{H}(r, \theta)} \text { is nonincreasing along any minimal geodesic } \\
& \text { segment from } p \text {. }
\end{aligned}
$$

Integrating (4.2) along the sphere directions, the radial direction gives the relative area and volume comparison, see e.g., [118].

Theorem 4.1 (Bishop-Gromov's Relative Volume Comparison). Suppose $M^{n}$ has $\operatorname{Ric}_{M} \geq(n-1) H$. Then

$$
\frac{\operatorname{Vol}(\partial B(p, r))}{\operatorname{Vol}_{H}(\partial B(r))} \text { and } \frac{\operatorname{Vol}(B(p, r))}{\operatorname{Vol}_{H}(B(r))} \text { are nonincreasing in } r \text {. }
$$

In particular,

$$
\begin{gathered}
\operatorname{Vol}(B(p, r)) \leq \operatorname{Vol}_{H}(B(r)) \quad \text { for all } r>0, \\
\frac{\operatorname{Vol}(B(p, r))}{\operatorname{Vol}(B(p, R))} \geq \frac{\operatorname{Vol}_{H}(B(r))}{\operatorname{Vol}_{H}(B(R))} \quad \text { for all } 0<r \leq R,
\end{gathered}
$$

and equality holds if and only if $B(p, r)$ is isometric to $B_{H}(r)$.

This is a powerful result because it is a global comparison. The volume of any ball is bounded above by the volume of the corresponding ball in the model, and if the volume of a big ball has a lower bound, then all smaller balls also have lower bounds. One can also apply the result to an annulus or a section of the directions. For topological applications see Section 6 . 
The volume element comparison (4.2) can also be used to prove a heat kernel comparison [33] and Cheeger-Colding's segment inequality [25, Theorem 2.11], see also [23].

Given a function $g \geq 0$ on $M^{n}$, put

$$
\mathcal{F}_{g}\left(x_{1}, x_{2}\right)=\inf _{\gamma} \int_{0}^{l} g(\gamma(s)) d s,
$$

where the inf is taken over all minimal geodesics $\gamma$ from $x_{1}$ to $x_{2}$ and $s$ denotes the arclength.

Theorem 4.2 (Segment Inequality, Cheeger-Colding 1996). Let Ric $_{M^{n}} \geq$ $-(n-1), A_{1}, A_{2} \subset B(p, r)$, and $r \leq R$. Then

$$
\int_{A_{1} \times A_{2}} \mathcal{F}_{g}\left(x_{1}, x_{2}\right) \leq c(n, R) \cdot r \cdot\left(\operatorname{Vol}\left(A_{1}\right)+\operatorname{Vol}\left(A_{2}\right)\right) \cdot \int_{B(p, 2 R)} g,
$$

where $c(n, R)=2 \sup _{0<\frac{s}{2} \leq u \leq s, 0<s<R} \frac{\operatorname{Vol}_{-1}(\partial B(s))}{\operatorname{Vol}_{-1}(\partial B(u))}$.

The segment inequality shows that if the integral of $g$ on a ball is small then the integral of $g$ along almost all segments is small. It also implies a Poincaré inequality of type $(1, p)$ for all $p \geq 1$ for manifolds with lower Ricci curvature bound [16]. In particular it gives a lower bound on the first eigenvalue of the Laplacian for the Dirichlet problem on a metric ball; compare [64].

The volume comparison theorem can be generalized to an integral Ricci lower bound $[\mathbf{8 9}]$, see also $[\mathbf{4 6}, \mathbf{1 1 5}]$. For convenience we introduce some notation.

For each $x \in M^{n}$ let $\lambda(x)$ denote the smallest eigenvalue for the Ricci tensor Ric : $T_{x} M \rightarrow T_{x} M$, and $\operatorname{Ric}_{-}^{H}(x)=((n-1) H-\lambda(x))_{+}=\max \{0$, $(n-1) H-\lambda(x)\}$. Let

$$
\left\|\operatorname{Ric}_{-}^{H}\right\|_{p}(R)=\sup _{x \in M}\left(\int_{B(x, R)}\left(\operatorname{Ric}_{-}^{H}\right)^{p} d v o l\right)^{\frac{1}{p}} .
$$

$\left\|\operatorname{Ric}_{-}^{H}\right\|_{p}$ measures the amount of Ricci curvature lying below $(n-1) H$ in the $L^{p}$ sense. Clearly $\left\|\operatorname{Ric}_{-}^{H}\right\|_{p}(R)=0$ iff $\operatorname{Ric}_{M} \geq(n-1) H$.

Parallel to the mean curvature comparison theorem (2.6) under pointwise Ricci curvature lower bound, Petersen-Wei [89] showed one can estimate the amount of mean curvature bigger than the mean curvature in the model by the amount of Ricci curvature lying below $H$ in $L^{p}$ sense. Namely for any $p>\frac{n}{2}, H \in \mathbb{R}$, and when $H>0$ assume $r \leq \frac{\pi}{2 \sqrt{H}}$, we have

$$
\left(\int_{B(x, r)}\left(m-m_{H}\right)_{+}^{2 p} d v o l\right)^{\frac{1}{2 p}} \leq C(n, p) \cdot\left(\left\|\operatorname{Ric}_{-}^{H}\right\|_{p}(r)\right)^{\frac{1}{2}}
$$


Using (4.8) we have

Theorem 4.3 (Relative Volume Estimate, Petersen-Wei 1997). Let $x \in M^{n}, H \in \mathbb{R}$ and $p>\frac{n}{2}$ be given; then there is a constant $C(n, p, H, R)$ which is nondecreasing in $R$ such that if $r \leq R$ and when $H>0$ assume that $R \leq \frac{\pi}{2 \sqrt{H}}$, we have

$$
\left(\frac{\operatorname{Vol} B(x, R)}{\operatorname{Vol}_{H}(B(R))}\right)^{\frac{1}{2 p}}-\left(\frac{\operatorname{Vol} B(x, r)}{\operatorname{Vol}_{H}(B(r))}\right)^{\frac{1}{2 p}} \leq C(n, p, H, R) \cdot\left(\left\|\operatorname{Ric}_{-}^{H}\right\|_{p}(R)\right)^{\frac{1}{2}} .
$$

Furthermore, when $r=0$ we obtain

$$
\operatorname{Vol} B(x, R) \leq\left(1+C(n, p, H, R) \cdot\left(\left\|\mathrm{Ric}_{-}^{H}\right\|_{p}(R)\right)^{\frac{1}{2}}\right)^{2 p} \operatorname{Vol}_{H}(B(R)) .
$$

Note that when $\left\|\operatorname{Ric}_{-}^{H}\right\|_{p}(R)=0$, this gives the Bishop-Gromov relative volume comparison.

Volume comparison is a powerful tool for studying manifolds with lower Ricci curvature bound and has many applications. As a result of (4.10), many results with pointwise Ricci lower bound (i.e., $\left\|\operatorname{Ric}_{-}^{H}\right\|_{p}(R)=0$ ) can be extended to the case when $\left\|\operatorname{Ric}_{-}^{H}\right\|_{p}(R)$ is very small $[\mathbf{4 6 , 8 9 , 8 8 , 4 0 ,}$ $104,90,41,8]$.

Perelman's reduced volume monotonicity [85], a basic and powerful tool in his work on Thurston's geometrization conjecture, is a generalization of Bishop-Gromov's volume comparison to Ricci flow. In fact Perelman gave a heuristic argument that volume comparison on an infinite dimensional space (incorporating the Ricci flow) gives the reduced volume monotonicity. It would be very interesting to investigate this relationship further.

\section{Rigidity results and stability}

From comparison theorems, various quantities are bounded by those of the model. When equality occurs one has the rigid case. In this section we concentrate on the rigidity and stability results for nonnegative and positive Ricci curvature. See Section 7 for rigidity and stability under GromovHausdorff convergence and a general lower bound.

The simplest rigidity is the maximal volume. From the equality of volume comparison (4.4), we deduce that if $M^{n}$ has $\operatorname{Ric}_{M} \geq n-1$ and $\operatorname{Vol}_{M}=$ $\operatorname{Vol}\left(S^{n}\right)$, then $M^{n}$ is isometric to $S^{n}$. Similarly if $M^{n}$ has $\operatorname{Ric}_{M} \geq 0$ and $\lim _{r \rightarrow \infty} \frac{\operatorname{Vol} B(p, r)}{\omega_{n} r^{n}}=1$, where $p \in M$ and $\omega_{n}$ is the volume of the unit ball in $\mathbb{R}^{n}$, then $M^{n}$ is isometric to $\mathbb{R}^{n}$.

From the equality of the area of a geodesic ball (the first quantity in (4.3)) we get another volume rigidity: volume annulus implies metric 
annulus. This is first observed in [25, Section 4], see also [24, Theorem 2.6]. For the case of nonnegative Ricci curvature, this result says that if $\operatorname{Ric}_{M^{n}} \geq 0$ on the annulus $A\left(p, r_{1}, r_{2}\right)$, and

$$
\frac{\operatorname{Vol}\left(\partial B\left(p, r_{1}\right)\right)}{\operatorname{Vol}\left(\partial B\left(p, r_{2}\right)\right)}=\frac{r_{1}^{n-1}}{r_{2}^{n-1}},
$$

then the metric on $A\left(p, r_{1}, r_{2}\right)$ is of the form $d r^{2}+r^{2} \tilde{g}$ for some smooth Riemannian metric $\tilde{g}$ on $\partial B\left(p, r_{1}\right)$.

By Myers' theorem (see Theorem 6.1), when Ricci curvature has a positive lower bound the diameter is bounded by the diameter of the model. In the maximal case, using an eigenvalue comparison (see below) Cheng [35] proved that if $M^{n}$ has $\operatorname{Ric}_{M} \geq n-1$ and $\operatorname{diam}_{M}=\pi$, then $M^{n}$ is isometric to $S^{n}$. This result can also be directly proven using volume comparison $[98,118]$.

The maximal diameter theorem for the noncompact case is given by Cheeger-Gromoll's splitting theorem [30]. The splitting theorem is the most important rigidity result. It plays a very important role in the study of manifolds with nonnegative Ricci curvature and manifolds with general Ricci lower bound.

TheOREM 5.1 (Splitting Theorem, Cheeger-Gromoll 1971). Let $M^{n}$ be a complete Riemannian manifold with $\operatorname{Ric}_{M} \geq 0$. If $M$ has a line, then $M$ is isometric to the product $\mathbb{R} \times N^{n-1}$, where $N$ is an $n-1$ dimensional manifold with $\operatorname{Ric}_{N} \geq 0$.

The result can be proven using the global Laplacian comparison (Theorem 3.6), the strong maximum principle (Theorem 3.5), the Bochner formula (2.1) and the de Rham decomposition theorem, see e.g., [118, 23, 86] for detail.

As an application of the splitting theorem we have that the first Betti number of $M$ is less than or equal to $n$ for $M^{n}$ with $\operatorname{Ric}_{M} \geq 0$, and $b_{1}=n$ if and only if $M$ is isometric to $T^{n}$ (the flat torus).

Applying the Bochner formula (2.1) to the first eigenfunction Lichnerowicz showed that if $M^{n}$ has $\operatorname{Ric}_{M} \geq n-1$, then the first eigenvalue $\lambda_{1}(M) \geq n[65]$. Obata showed that if $\lambda_{1}(M)=n$ then $M^{n}$ is isometric to $S^{n}[79]$.

From these rigidity results (the equal case), we naturally ask what happens in the almost equal case. Many results are known in this case. For volume we have the following beautiful stability results for positive and nonnegative Ricci curvatures [26].

Theorem 5.2 (Volume Stability, Cheeger-Colding, 1997). There exists $\epsilon(n)>0$ such that

(i) if a complete Riemannian manifold $M^{n}$ has $\operatorname{Ric}_{M} \geq n-1$ and $\operatorname{Vol}_{M} \geq(1-\epsilon(n)) \operatorname{Vol}\left(S^{n}\right)$, then $M^{n}$ is diffeomorphic to $S^{n}$; 
(ii) if a complete Riemannian manifold $M^{n}$ has $\operatorname{Ric}_{M} \geq 0$ and for some $p \in M, \operatorname{Vol} B(p, r) \geq(1-\epsilon(n)) \omega_{n} r^{n}$ for all $r>0$, then $M^{n}$ is diffeomorphic to $\mathbb{R}^{n}$.

These were first proved by Perelman [82] with the weaker conclusion that (i) $M^{n}$ is homeomorphic to $S^{n}$, (ii) $M^{n}$ is contractible.

The analogous stability result is not true for diameter. In fact, there are manifolds with Ric $\geq n-1$ and diameter arbitrarily close to $\pi$ which are not homotopic to sphere $[\mathbf{3}, \mathbf{8 0}]$. This should be contrasted with the sectional curvature case, where we have the beautiful Grove-Shiohama diameter sphere theorem [54], that if $M^{n}$ has sectional curvature $K_{M} \geq 1$ and $\operatorname{diam}_{M}>\pi / 2$ then $M$ is homeomorphic to $S^{n}$. Anderson showed that the stability for the splitting theorem (Theorem 5.1) does not hold either [6].

By work of Cheng and Croke $[\mathbf{3 5}, \mathbf{3 9}]$, if $\operatorname{Ric}_{M} \geq n-1$ then $\operatorname{diam}_{M}$ is close to $\pi$ if and only if $\lambda_{1}(M)$ is close to $n$. So the naive version of the stability for $\lambda_{1}(M)$ does not hold either. However, from the work of $[\mathbf{3 6 , 2 6 , 8 7}$ we have the following modified version.

Theorem 5.3 (Colding, Cheeger-Colding, Petersen). There exists $\epsilon(n)$ $>0$ such that if a complete Riemannian manifold $M^{n}$ has $\operatorname{Ric}_{M} \geq n-1$, and radius $\geq \pi-\epsilon(n)$ or $\lambda_{n+1}(M) \leq n+\epsilon(n)$, then $M^{n}$ is diffeomorphic to $S^{n}$.

Here $\lambda_{n+1}(M)$ is the $(n+1)$-th eigenvalue of the Laplacian. The above condition is natural in the sense that for $S^{n}$ the radius is $\pi$ and the first eigenvalue is $n$ with multiplicity $n+1$. Extending Cheng and Croke's work Petersen showed that if $\operatorname{Ric}_{M} \geq n-1$ then the radius is close to $\pi$ if and only if $\lambda_{n+1}(M)$ is close to $n$.

The stability for the first Betti number, conjectured by Gromov, was proved by Cheeger-Colding in [26]. Namely there exists $\epsilon(n)>0$ such that if a complete Riemannian manifold $M^{n}$ has $\operatorname{Ric}_{M}\left(\operatorname{diam}_{M}\right)^{2} \geq-\epsilon(n)$ and $b_{1}=n$, then $M$ is diffeomorphic to $T^{n}$. The homeomorphic version was first proved in $[\mathbf{3 7}]$.

Although the direct stability for diameter does not hold, CheegerColding's breakthrough work [25] gives quantitative generalizations of the diameter rigidity results, see Section 8.

\section{The fundamental groups}

In lower dimensions $(n \leq 3)$ a Ricci curvature lower bound has strong topological implications. R. Hamilton [56] proved that compact manifolds $M^{3}$ with positive Ricci curvature are space forms. Schoen-Yau [92] proved that any complete open manifold $M^{3}$ with positive Ricci curvature must be diffeomorphic to $\mathbb{R}^{3}$ using minimal surfaces. In general the strongest control is on the fundamental group.

The first result is Myers' theorem [76]. 
Theorem 6.1 (Myers, 1941). If $\operatorname{Ric}_{M} \geq H>0$, then $\operatorname{diam}(M) \leq \pi / \sqrt{H}$, and $\pi_{1}(M)$ is finite.

This is the only known topological obstruction to a compact manifold that supports a metric with positive Ricci curvature other than topological obstructions shared by manifolds with positive scalar curvature. See Section 10 for examples with positive Ricci curvature and Rosenberg's article in this volume for a discussion of scalar curvature.

We can still ask what one can say about the finite group. Any finite group can be realized as the fundamental group of a compact manifold with positive Ricci curvature since any finite group is a subgroup of $S U(n)$ (for $\mathrm{n}$ sufficiently big) and $S U(n)$ has a metric with positive Ricci curvature (in fact Einstein). What can one say if the dimension $n$ is fixed? For example, is the order of the group modulo an abelian subgroup bounded by the dimension? See $[\mathbf{1 0 9}]$ for a partial result.

For a compact manifold $M$ with nonnegative Ricci curvature, CheegerGromoll's splitting theorem (Theorem 5.1) implies that $\pi_{1}(M)$ has an abelian subgroup of finite index [30]. Again it is open if one can bound the index by dimension.

For general nonnegative Ricci curvature manifolds, using covering and volume comparison Milnor showed that [75]

THEOREM 6.2 (Milnor, 1968). If $M^{n}$ is complete with $\operatorname{Ric}_{M} \geq 0$, then any finitely generated subgroup of $\pi_{1}(M)$ has polynomial growth of degree $\leq n$.

Combining this with the following result of Gromov [51], we know that any finitely generated subgroup of $\pi_{1}(M)$ of manifolds with nonnegative Ricci curvature is almost nilpotent.

TheOREM 6.3 (Gromov, 1981). A finitely generated group $\Gamma$ has polynomial growth iff $\Gamma$ is almost nilpotent, i.e., it contains a nilpotent subgroup of finite index.

When $M^{n}$ has nonnegative Ricci curvature and Euclidean volume growth (i.e., $\operatorname{Vol} B(p, r) \geq c r^{n}$ for some $c>0$ ), using a heat kernel estimate Li showed that $\pi_{1}(M)$ is finite [63]. Anderson also derived this using volume comparison [4]. Using the splitting theorem of Cheeger and Gromoll [30] (Theorem 5.1) on the universal cover Sormani showed that a noncompact manifold with positive Ricci curvature has the loops-to-infinity property [99]. As a consequence she showed that a noncompact manifold with positive Ricci curvature is simply connected if it is simply connected at infinity. See $[\mathbf{9 6}, \mathbf{1 1 3}]$ for more applications of the loops-to-infinity property.

From the above one naturally wonders if all nilpotent groups occur as the fundamental group of a complete non-compact manifold with nonnegative Ricci curvature. Indeed, extending the warping product constructions 
in $[\mathbf{7 7}, \mathbf{1 1}]$, Wei showed $[\mathbf{1 0 5}]$ that any finitely generated torsion free nilpotent group could occur as fundamental group of a manifold with positive Ricci curvature. Wilking [109] extended this to any finitely generated almost nilpotent group. This gives a very good understanding of the fundamental group of a manifold with nonnegative Ricci curvature except the following long standing problem regarding the finiteness of generators [75].

Conjecture 6.4 (Milnor, 1968). The fundamental group of a manifold with nonnegative Ricci curvature is finitely generated.

There is some very good progress in this direction. Using short generators and a uniform cut lemma based on the excess estimate of Abresch and Gromoll [1] (see (8.2)) Sormani [101] proved that if $\operatorname{Ric}_{M} \geq 0$ and $M^{n}$ has small linear diameter growth, then $\pi_{1}(M)$ is finitely generated. More precisely the small linear growth condition is:

$$
\limsup _{r \rightarrow \infty} \frac{\operatorname{diam} \partial B(p, r)}{r}<s_{n}=\frac{n}{(n-1) 3^{n}}\left(\frac{n-1}{n-2}\right)^{n-1} .
$$

The constant $s_{n}$ was improved in $[\mathbf{1 1 4}]$. Then in [112] Wylie proved that in this case $\pi_{1}(M)=G(r)$ for $r$ big, where $G(r)$ is the image of $\pi_{1}(B(p, r))$ in $\pi_{1}(B(p, 2 r))$. In an earlier paper [100], Sormani proved that all manifolds with nonnegative Ricci curvature and linear volume growth have sublinear diamter growth, so manifolds with linear volume growth are covered by these results. Any open manifold with nonnegative Ricci curvature has at least linear volume growth $[\mathbf{1 1 6}]$.

In a very different direction Wilking [109], using algebraic methods, showed that if $\operatorname{Ric}_{M} \geq 0$ then $\pi_{1}(M)$ is finitely generated iff any abelian subgroup of $\pi_{1}(M)$ is finitely generated, effectively reducing the Milnor conjecture to the study of manifolds with abelian fundamental groups.

The fundamental group and the first Betti number are very nicely related. So it is natural that Ricci lower bound also controls the first Betti number. For compact manifolds Gromov [52] and Gallot [45] showed that if $M^{n}$ is a compact manifold with

$$
\operatorname{Ric}_{M} \geq(n-1) H, \quad \operatorname{diam}_{M} \leq D,
$$

then there is a function $C\left(n, H D^{2}\right)$ such that $b_{1}(M) \leq C\left(n, H D^{2}\right)$ and $\lim _{x \rightarrow 0^{-}} C(n, x)=n$ and $C(n, x)=0$ for $x>0$. In particular, if $H D^{2}$ is small, $b_{1}(M) \leq n$.

The celebrated Betti number estimate of Gromov [50] shows that all higher Betti numbers can be bounded by sectional curvature and diameter. This is not true for Ricci curvature. Using semi-local surgery Sha-Yang constructed metrics of positive Ricci curvature on the connected sum of $k$ copies of $S^{2} \times S^{2}$ for all $k \geq 1$ [95]. Recently, using Seifert bundles over orbifolds with a Kähler Einstein metric, Kollar showed that there are Einstein metrics 
with positive Ricci curvature on the connected sums of arbitrary number of copies of $S^{2} \times S^{3}[\mathbf{6 1}$.

Kapovitch-Wilking [59] recently announced a proof of the compact analog of Milnor's conjecture that the fundamental group of a manifold satisfying (6.1) has a presentation with a universally bounded number of generators (as conjectured by this author), and that a manifold which admits almost nonnegative Ricci curvature has a virtually nilpotent fundamental group. The second result would greatly generalize Fukaya-Yamaguchi's work on almost nonnegative sectional curvature $[\mathbf{4 4}]$. See $[\mathbf{1 0 6}, \mathbf{1 0 7}]$ for earlier partial results.

When the volume is also bounded from below, by using a clever covering argument M. Anderson [5] showed that the number of the short homotopically nontrivial closed geodesics can be controlled and for the class of manifolds $M$ with $\operatorname{Ric}_{M} \geq(n-1) H, \operatorname{Vol}_{M} \geq V$ and $\operatorname{diam}_{M} \leq D$ there are only finitely many isomorphism types of $\pi_{1}(M)$. Again, if the Ricci curvature is replaced by sectional curvature then much more can be said. Namely there are only finitely many homeomorphism types of the manifolds with sectional curvature and volume bounded from below and diameter bounded from above $[\mathbf{5 3}, \mathbf{8 1}]$. By $[\mathbf{8 4}]$ this is not true for Ricci curvature unless the dimension is 3 [117].

Contrary to a Ricci curvature lower bound, a Ricci curvature upper bound does not have any topological constraint [68].

THEOREM 6.5 (Lohkamp, 1994). If $n \geq 3$, any manifold $M^{n}$ admits a complete metric with $\operatorname{Ric}_{M}<0$.

An upper Ricci curvature bound does have geometric implications, e.g., the isometry group of a compact manifold with negative Ricci curvature is finite. In the presence of a lower bound, an upper bound on Ricci curvature forces additional regularity of the metric, see Theorem 9.8 in Section 9 by Anderson. It's still unknown whether it will give additional topological control. For example, the following question is still open.

Question 6.6. Does the class of manifolds $M^{n}$ with $\left|\operatorname{Ric}_{M}\right| \leq H, \operatorname{Vol}_{M}$ $\geq V$ and $\operatorname{diam}_{M} \leq D$ have finite many homotopy types?

There are infinitely many homotopy types without the Ricci upper bound $[\mathbf{8 4}]$.

\section{Gromov-Hausdorff convergence}

Gromov-Hausdorff convergence is very useful in studying manfolds with a lower Ricci bound. The starting point is Gromov's precompactness theorem. Let's first recall the Gromov-Hausdorff distance. See [52, Chapter 3,5], [86, Chapter 10], [15, Chapter 7] for more background material on Gromov-Hausdorff convergence. 
Given a metric space $(X, d)$ and subsets $A, B \subset X$, the Hausdorff distance is

$$
d_{H}(A, B)=\inf \left\{\epsilon>0: B \subset T_{\epsilon}(A) \text { and } A \subset T_{\epsilon}(B)\right\},
$$

where $T_{\epsilon}(A)=\{x \in X: d(x, A)<\epsilon\}$.

Definition 7.1 (Gromov, 1981). Given two compact metric spaces $X, Y$, the Gromov-Hausdorff distance is $d_{G H}(X, Y)=\inf \left\{d_{H}(X, Y)\right.$ : all metrics on the disjoint union, $X \coprod Y$, which extend the metrics of $X$ and $Y$ \}.

The Gromov-Hausdorff distance defines a metric on the collection of isometry classes of compact metric spaces. Thus, there is the naturally associated notion of Gromov-Hausdorff convergence of compact metric spaces. While the Gromov-Hausdorff distance makes sense for non-compact metric spaces, the following looser definition of convergence is more appropriate. See also [52, Defn 3.14]. These two definitions are equivalent [103, Appendix].

Definition 7.2. We say that non-compact metric spaces $\left(X_{i}, x_{i}\right)$ converge in the pointed Gromov-Hausdorff sense to $(Y, y)$ if for any $r>0$, $B\left(x_{i}, r\right)$ converges to $B(y, r)$ in the pointed Gromov-Hausdorff sense.

Applying the relative volume comparison (4.5) to manifolds with lower Ricci curvature bound, we have

TheOREM 7.3 (Gromov's precompactness theorem). The class of closed manifolds $M^{n}$ with $\operatorname{Ric}_{M} \geq(n-1) H$ and $\operatorname{diam}_{M} \leq D$ is precompact. The class of pointed complete manifolds $M^{n}$ with $\operatorname{Ric}_{M} \geq(n-1) H$ is precompact.

By the above, for an open manifold $M^{n}$ with $\operatorname{Ric}_{M} \geq 0$ any sequence $\left\{\left(M^{n}, x, r_{i}^{-2} g\right)\right\}$, with $r_{i} \rightarrow \infty$, subconverges in the pointed GromovHausdorff topology to a length space $M_{\infty}$. In general, $M_{\infty}$ is not unique [83]. Any such limit is called an asymptotic cone of $M^{n}$, or a cone of $M^{n}$ at infinity .

Gromov-Hausdorff convergence defines a very weak topology. In general one only knows that Gromov-Hausdorff limit of length spaces is a length space and diameter is continuous under the Gromov-Hausdorff convergence. When the limit is a smooth manifold with same dimension Colding showed the remarkable result that for manifolds with lower Ricci curvature bound the volume also converges [37], which was conjectured by Anderson-Cheeger. See also [23] for a proof using mod 2 degree.

Theorem 7.4 (Volume Convergence, Colding, 1997). If $\left(M_{i}^{n}, x_{i}\right)$ has $\operatorname{Ric}_{M_{i}} \geq(n-1) H$ and converges in the pointed Gromov-Hausdorff sense to smooth Riemannian manifold $\left(M^{n}, x\right)$, then for all $r>0$

$$
\lim _{i \rightarrow \infty} \operatorname{Vol}\left(B\left(x_{i}, r\right)\right)=\operatorname{Vol}(B(x, r)) .
$$


The volume convergence can be generalized to the noncollapsed singular limit space (by replacing the Riemannian volume with the $n$-dimensional Hausdorff measure $\mathcal{H}^{n}$ ) [26, Theorem 5.9], and to the collapsing case with smooth limit $M^{k}$ in terms of the $k$-dimensional Hausdorff content $[\mathbf{2 7}$, Theorem 1.39].

As an application of Theorem 7.4, Colding [37] derived the rigidity result that if $M^{n}$ has $\operatorname{Ric}_{M} \geq 0$ and some $M_{\infty}$ is isometric to $\mathbb{R}^{n}$, then $M$ is isometric to $\mathbb{R}^{n}$.

We also have the following wonderful stability result [26] which sharpens an earlier version in [37].

ThEOREM 7.5 (Cheeger-Colding, 1997). For a closed Riemannian manifold $M^{n}$ there exists an $\epsilon(M)>0$ such that if $N^{n}$ is a n-manifold with $\operatorname{Ric}_{N} \geq-(n-1)$ and $d_{G H}(M, N)<\epsilon$ then $M$ and $N$ are diffeomorphic.

Unlike the sectional curvature case, examples show that the result does not hold if one allows $M$ to have singularities even on the fundamental group level [80, Remark (2)]. Also the $\epsilon$ here must depend on $M[\mathbf{3}]$.

Cheeger-Colding also showed that the eigenvalues and eigenfunctions of the Laplacian are continuous under measured Gromov-Hausdorff convergence [28]. To state the result we need a definition and some structure result on the limit space (see Section 9 for more structures). Let $X_{i}$ be a sequence of metric spaces converging to $X_{\infty}$ and $\mu_{i}, \mu_{\infty}$ be Radon measures on $X_{i}, X_{\infty}$.

Definition 7.6. We say $\left(X_{i}, \mu_{i}\right)$ converges in the measured GromovHausdorff sense to $\left(X_{\infty}, \mu_{\infty}\right)$ if for all sequences of continuous functions $f_{i}: X_{i} \rightarrow \mathbb{R}$ converging to $f_{\infty}: X_{\infty} \rightarrow \mathbb{R}$, we have

$$
\int_{X_{i}} f_{i} d \mu_{i} \rightarrow \int_{X_{\infty}} f_{\infty} d \mu_{\infty} .
$$

If $\left(M_{\infty}, p\right)$ is the pointed Gromov-Hausdorff limit of a sequence of Riemannian manifolds $\left(M_{i}^{n}, p_{i}\right)$ with $\operatorname{Ric}_{M_{i}} \geq-(n-1)$, then there is a natural collection of measures, $\mu$, on $M_{\infty}$ obtained by taking limits of the normalized Reimannian measures on $M_{j}^{n}$ for a suitable subsequence $M_{j}^{n}$ [43], [26, Section 1],

$$
\mu=\lim _{j \rightarrow \infty} \underline{\operatorname{Vol}}_{j}(\cdot)=\operatorname{Vol}(\cdot) / \operatorname{Vol}\left(B\left(p_{j}, 1\right)\right)
$$

In particular, for all $z \in M_{\infty}$ and $0<r_{1} \leq r_{2}$, we have the renormalized limit measure $\mu$ satisfy the following comparison

$$
\frac{\mu\left(B\left(z, r_{1}\right)\right)}{\mu\left(B\left(z, r_{2}\right)\right)} \geq \frac{\operatorname{Vol}_{n,-1}\left(B\left(r_{1}\right)\right)}{\operatorname{Vol}_{n,-1}\left(B\left(r_{2}\right)\right)} .
$$

With this, the extension of the segment inequality (4.6) to the limit, the gradient estimate (2.8), and Bochner's formula, one can define a canonical 
self-adjoint Laplacian $\Delta_{\infty}$ on the limit space $M_{\infty}$ by means of limits of the eigenfunctions and eigenvalues for the sequence of the manifolds. In $[\mathbf{1 9}, \mathbf{2 8}]$ an intrinsic construction of this operator is also given on more general metric measure spaces. Let $\left\{\lambda_{1, i} \cdots,\right\},\left\{\lambda_{1, \infty}, \cdots,\right\}$ denote the eigenvalues for $\Delta_{i}, \Delta_{\infty}$ on $M_{i}, M_{\infty}$, and $\phi_{j, i}, \phi_{j, \infty}$ the eigenfunctions of the jth eigenvalues $\lambda_{j, i}, \lambda_{j, \infty}$. In [28] Cheeger-Colding in particular proved the following theorem, establishing Fukaya's conjecture [43].

Theorem 7.7 (Spectral Convergence, Cheeger-Colding, 2000). Let $\left(M_{i}^{n}, p_{i}, \mathrm{Vol}_{i}\right)$ with $\operatorname{Ric}_{M_{i}} \geq-(n-1)$ converge to $\left(M_{\infty}, p, \mu\right)$ under measured Gromov-Hausdorff sense and $M_{\infty}$ be compact. Then for each $j, \lambda_{j, i} \rightarrow \lambda_{j, \infty}$ and $\phi_{j, i} \rightarrow \phi_{j, \infty}$ uniformly as $i \rightarrow \infty$.

As a natural extension, in [42] Ding proved that the heat kernel and Green's function also behave nicely under the measured Gromov-Hausdorff convergence. The natural extension to the $p$-form Laplacian does not hold; however, there is still very nice work in this direction by John Lott, see $[69,71]$.

\section{Almost rigidity and applications}

Although the analogous stability results for maximal diameter in the case of positive/nonnegative Ricci curvature do not hold, Cheeger-Colding's significant work [25] provides quantitative generalizations of Cheng's maximal diameter theorem and Cheeger-Gromoll's splitting theorem (Theorem 5.1), and the volume annulus implies the metric annulus theorem in terms of Gromov-Hausdroff distance. These results have important applications in extending rigidity results to the limit space.

An important ingredient for these results is Abresch-Gromoll's excess estimate [1]. For $y_{1}, y_{2} \in M^{n}$, the excess function $E$ with respect to $y_{1}, y_{2}$ is

$$
E_{y_{1}, y_{2}}(x)=d\left(y_{1}, x\right)+d\left(y_{2}, x\right)-d\left(y_{1}, y_{2}\right) .
$$

Clearly $E$ is Lipschitz with Lipschitz constant $\leq 2$.

Let $\gamma$ be a minimal geodesic from $y_{1}$ to $y_{2}, s(x)=\min \left(d\left(y_{1}, x\right), d\left(y_{2}, x\right)\right)$ and $h(x)=\min _{t} d(x, \gamma(t))$, the height from $x$ to a minimal geodesic $\gamma(t)$ connecting $y_{1}$ and $y_{2}$. By the triangle inequality $0 \leq E(x) \leq 2 h(x)$. Applying the Laplacian comparison (Theorem 3.6) to $E(x)$ and with an elaborate (quantitative) use of the maximum principle (Theorem 3.5) Abresch-Gromoll showed that if $\operatorname{Ric}_{M} \geq 0$ and $h(x) \leq \frac{s(x)}{2}$, then ([1] , see also [22])

$$
E(x) \leq 4\left(\frac{h^{n}}{s}\right)^{\frac{1}{n-1}} .
$$

This is the first distance estimate in terms of a lower Ricci curvature bound.

The following version (not assuming $E(p)=0$, but without the sharp estimate) is from [23, Theorem 9.1]. 
ThEOREM 8.1 (Excess Estimate, Abresch-Gromoll, 1990). If $M^{n}$ has $\operatorname{Ric}_{M} \geq-(n-1) \delta$, and for $p \in M, s(p) \geq L$ and $E(p) \leq \epsilon$, then on $B(p, R)$, $E \leq \Psi=\Psi\left(\delta, L^{-1}, \epsilon \mid n, R\right)$, where $\Psi$ is a nonnegative constant such that for fixed $n$ and $R \Psi$ goes to zero as $\delta, \epsilon \rightarrow 0$ and $L \rightarrow \infty$.

This can be interpreted as a weak almost splitting theorem. CheegerColding generalized this result tremendously by proving the following almost splitting theorem $[\mathbf{2 5}]$, see also [23].

Theorem 8.2 (Almost Splitting, Cheeger-Colding, 1996). With the same assumptions as Theorem 8.1, there is a length space $X$ such that for some ball, $B\left((0, x), \frac{1}{4} R\right) \subset \mathbb{R} \times X$, with the product metric, we have

$$
d_{G H}\left(B\left(p, \frac{1}{4} R\right), B\left((0, x), \frac{1}{4} R\right)\right) \leq \Psi .
$$

Note that $X$ here may not be smooth, and the Hausdorff dimension could be smaller than $n-1$. Examples also show that the ball $B\left(p, \frac{1}{4} R\right)$ may not have the topology of a product, no matter how small $\delta, \epsilon$, and $L^{-1}$ are $[6,73]$.

The proof is quite involved. Using the Laplacian comparison, the maximum principle, and Theorem 8.1 one shows that the distance function $b_{i}=$ $d\left(x, y_{i}\right)-d\left(p, y_{i}\right)$ associated to $p$ and $y_{i}$ is uniformly close to $\mathbf{b}_{i}$, the harmonic function with same values on $\partial B(p, R)$. From this, together with the lower bound for the smallest eigenvalue of the Dirichlet problem on $B(p, R)$ (see Theorem 4.2) one shows that $\nabla b_{i}, \nabla \mathbf{b}_{i}$ are close in the $L_{2}$ sense. In particular $\nabla \mathbf{b}_{i}$ is close to 1 in the $L_{2}$ sense. Then applying the Bochner formula to $\mathbf{b}_{i}$ multiplied with a cut-off function with bounded Laplacian one shows that $\left|\operatorname{Hess}_{i}\right|$ is small in the $L_{2}$ sense in a smaller ball. Finally, in the most significant step, by using the segment inequality (4.6), the gradient estimate (2.7) and the information established above one derives a quantitative version of the Pythagorean theorem, showing that the ball is close in the Gromov-Hausdorff sense to a ball in some product space; see $[\mathbf{2 5}, \mathbf{2 3}]$.

An immediate application of the almost splitting theorem is the extension of the splitting theorem to the limit space.

TheOREM 8.3 (Cheeger-Colding, 1996). If $M_{i}^{n}$ has $\operatorname{Ric}_{M_{i}} \geq-(n-1) \delta_{i}$ with $\delta_{i} \rightarrow 0$ as $i \rightarrow \infty$, converges to $Y$ in the pointed Gromov-Hausdorff sense, and $Y$ contains a line, then $Y$ is isometric to $\mathbb{R} \times X$ for some length space $X$.

Similarly, one has almost rigidity in the presence of finite diameter (with a simpler a proof) [25, Theorem 5.12]. As a special consequence, we have that if $M_{i}^{n}$ has $\operatorname{Ric}_{M_{i}} \geq(n-1)$, diam $M_{i} \rightarrow \pi$ as $i \rightarrow \infty$, and converges to $Y$ in the Gromov-Hausdorff sense, then $Y$ is isometric to the spherical metric suspension of some length space $X$ with $\operatorname{diam}(X) \leq \pi$. This is a kind of stability for diameter. 
Along the same lines (with more complicated technical details) Cheeger and Colding [25] have an almost rigidity version for the volume annulus implies metric annulus theorem (see Section 5). As a very nice application to the asymptotic cone, they showed that if $M^{n}$ has $\operatorname{Ric}_{M} \geq 0$ and has Euclidean volume growth, then every asymptotic cone of $M$ is a metric cone.

\section{The structure of limit spaces}

As we have seen, understanding the structure of the limit space of manifolds with lower Ricci curvature bound often helps in understanding the structure of the sequence. Cheeger-Colding made significant progress in understanding the regularity and geometric structure of the limit spaces $[\mathbf{2 6}, \mathbf{2 7}, \mathbf{2 8}]$. On the other hand, Menguy constructed examples showing that the limit space could have infinite topology in an arbitrarily small neighborhood $[\mathbf{7 3}]$. In $[\mathbf{1 0 2}, \mathbf{1 0 3}]$ Sormani-Wei showed that the limit space has a universal cover.

Let $\left(Y^{m}, y\right)$ (Hausdorff dimension $m$ ) be the pointed Gromov-Hausdorff limit of a sequence of Riemannian manifolds $\left(M_{i}^{n}, p_{i}\right)$ with $\operatorname{Ric}_{M_{i}} \geq-(n-1)$. Then $m \leq n$ and $Y^{m}$ is locally compact. Moreover Cheeger-Colding [26] showed that if $m=\operatorname{dim} Y<n$, then $m \leq n-1$.

The basic notion for studying the infinitesimal structure of the limit space $Y$ is that of a tangent cone.

Definition 9.1. A tangent cone, $Y_{y}$, at $y \in\left(Y^{m}, d\right)$ is the pointed GromovHausdorff limit of a sequence of the rescaled spaces $\left(Y^{m}, r_{i} d, y\right)$, where $r_{i} \rightarrow \infty$ as $i \rightarrow \infty$.

By Gromov's precompactness theorem (Theorem 7.3), every such sequence has a converging subsequence. So tangent cones exist for all $y \in Y^{m}$, but might depend on the choice of convergent sequence. Clearly if $M^{n}$ is a Riemannian manifold, then the tangent cone at any point is isometric to $\mathbb{R}^{n}$. Motivated by this one defines [26]

Definition 9.2. A point, $y \in Y$, is called $k$-regular if for some $k$, every tangent cone at $y$ is isometric to $\mathbb{R}^{k}$. Let $\mathcal{R}_{k}$ denote the set of $k$-regular points and $\mathcal{R}=\cup_{k} \mathcal{R}_{k}$, the regular set. The singular set, $Y \backslash \mathcal{R}$, is denoted $\mathcal{S}$.

Let $\mu$ be a renormalized limit measure on $Y$ as in (7.3). Cheeger-Colding showed that the regular points have full measure $[\mathbf{2 6}]$.

TheOREM 9.3 (Cheeger-Colding, 1997). For any renormalized limit measure $\mu, \mu(\mathcal{S})=0$, in particular, the regular points are dense.

Furthermore, up to a set of measure zero, $Y$ is a countable union of sets, each of which is bi-Lipschitz equivalent to a subset of Euclidean space [28]. 
Definition 9.4. A metric measure space, $(X, \mu)$, is called $\mu$-rectifiable if $0<\mu(X)<\infty$, and there exist $N<\infty$ and a countable collection of subsets, $A_{j}$, with $\mu\left(X \backslash \cup_{j} A_{j}\right)=0$, such that each $A_{j}$ is bi-Lipschitz equivalent to a subset of $\mathbb{R}^{l(j)}$, for some $1 \leq l(j) \leq N$. In addtion, on the sets $A_{j}$, the measures $\mu$ and and the Hausdorff measure $\mathcal{H}^{l(j)}$ are mutually absolutely continous.

TheOREm 9.5 (Cheeger-Colding, 2000). Bounded subsets of $Y$ are $\mu$ rectifiable with respect to any renormalized limit measure $\mu$.

At the singular points, the structure could be very complicated. Following a related earlier construction of Perelman [84], Menguy constructed 4-dimensional examples of (noncollapsed) limit spaces with $\operatorname{Ric}_{M_{i}^{n}}>1$, for which there exists a point so that any neighborhood of the point has infinite second Betti number $[\mathbf{7 3}]$. See $[\mathbf{2 6}, \mathbf{7 2}, \mathbf{7 4}]$ for examples of collapsed limit space with interesting properties.

Although we have very good regularity results, not much topological structure is known for the limit spaces in general. E.g., is $Y$ locally simply connected? Although this is unknown, using the renormalized limit measure and the existence of regular points, together with $\delta$-covers, Sormani-Wei $[\mathbf{1 0 2}, \mathbf{1 0 3}]$ showed that the universal cover of $Y$ exists. Moreover when $Y$ is compact, the fundamental group of $M_{i}$ has a surjective homomorphism onto the group of deck transforms of $Y$ for all $i$ sufficiently large.

When the sequence has the additional assumption that

$$
\operatorname{Vol}\left(B\left(p_{i}, 1\right)\right) \geq v>0,
$$

the limit space $Y$ is called noncollapsed. This is equivalent to $m=n$. In this case, more structure is known.

Definition 9.6. Given $\epsilon>0$, the $\epsilon$-regular set, $\mathcal{R}_{\epsilon}$, consists of those points $y$ such that for all sufficiently small $r$,

$$
d_{G H}(B(y, r), B(0, r)) \leq \epsilon r
$$

where $0 \in \mathbb{R}^{n}$.

Clearly $\mathcal{R}=\cap_{\epsilon} \mathcal{R}_{\epsilon}$. Let $\stackrel{\circ}{\mathcal{R}} \epsilon$ denote the interior of $\mathcal{R}_{\epsilon}$.

TheOREM 9.7 (Cheeger-Colding 1997, 2000). There exists $\epsilon(n)>0$ such that if $Y$ is a noncollapsed limit space of the sequence $M_{i}^{n}$ with $\operatorname{Ric}_{M_{i}} \geq$ $-(n-1)$, then for $0<\epsilon<\epsilon(n)$, the set $\stackrel{\circ}{\mathcal{R}} \epsilon$ is $\alpha(\epsilon)$-bi-Hölder equivalent to a smooth connected Riemannian manifold, where $\alpha(\epsilon) \rightarrow 1$ as $\epsilon \rightarrow 0$. Moreover,

$$
\operatorname{dim}\left(Y \backslash \stackrel{\circ}{\mathcal{R}}_{\epsilon}\right) \leq n-2
$$


In addition, for all $y \in Y$, every tangent cone $Y_{y}$ at $y$ is a metric cone and the isometry group of $Y$ is a Lie group.

This is proved in $[\mathbf{2 6 , 2 7}$.

If, in addition, Ricci curvature is bounded from two sides, we have stronger regularity $[\mathbf{2}]$.

TheOREm 9.8 (Anderson, 1990). There exists $\epsilon(n)>0$ such that if $Y$ is a noncollapsed limit space of the sequence $M_{i}^{n}$ with $\left|\operatorname{Ric}_{M_{i}}\right| \leq n-1$, then for $0<\epsilon<\epsilon(n), \mathcal{R}_{\epsilon}=\mathcal{R}$. In particular the singular set is closed. Moreover, $\mathcal{R}$ is a $C^{1, \alpha}$ Riemannian manifold, for all $\alpha<1$. If the metrics on $M_{i}^{n}$ are Einstein, $\operatorname{Ric}_{M_{i}^{n}}=(n-1) H g_{i}$, then the metric on $\mathcal{R}$ is actually $C^{\infty}$.

Many more regularity results are obtained when the sequence is Einstein, Kähler, has special holonomy, or has bounded $L^{p}$-norm of the full curvature tensor; see $[\mathbf{7}, \mathbf{2 0}, \mathbf{2 1}, \mathbf{3 1}$, especially $[\mathbf{2 4}]$ which gives an excellent survey in this direction. See the recent work $[\mathbf{3 2}]$ for Einstein 4-manifolds with possible collapsing.

\section{Examples of manifolds with nonnegative Ricci curvature}

Many examples of manifolds with nonnegative Ricci curvature have been constructed, which contribute greatly to the study of manifolds with lower Ricci curvature bound. We only discuss the examples related to the basic methods here, therefore many specific examples are unfortunately omitted (some are mentioned in the previous sections). There are mainly three methods: fiber bundle construction, special surgery, and group quotient, all combined with warped products. These method are also very useful in constructing Einstein manifolds. A large class of Einstein manifolds is also provided by Yau's solution of the Calabi conjecture.

Note that if two compact Riemannian manifolds $M^{m}, N^{n}(n, m \geq 2)$ have positive Ricci curvature, then their product has positive Ricci curvature, which is not true for sectional curvature but only needs one factor positive for scalar curvature. Therefore it is natural to look at the fiber bundle case. Using Riemannian submersions with totally geodesic fibers J. C. Nash [78], W. A. Poor [91], and Berard-Bergery [10] showed that the compact total space of a fiber bundle admits a metric of positive Ricci curvature if the base and the fiber admit metrics with positive Ricci curvature and if the structure group acts by isometries. Furthermore, any vector bundle of rank $\geq 2$ over a compact manifold with Ric $>0$ carries a complete metric with positive Ricci curvature. In [48] Gilkey-Park-Tuschmann showed that a principal bundle $P$ over a compact manifold with Ric $>0$ and compact connected structure group $G$ admits a $G$ invariant metric with positive Ricci curvature if and only if $\pi_{1}(P)$ is finite. Unlike the product case, the corresponding statements for Ric $\geq 0$ are not true in all these cases, e.g., the nilmanifold $S^{1} \rightarrow N^{3} \rightarrow T^{2}$ does not admit a metric with Ric $\geq 0$. On the other hand Belegradek-Wei 
[9] showed that it is true in the stable sense. Namely, if $E$ is the total space of a bundle over a compact base with $\mathrm{Ric} \geq 0$, and has either a compact Ric $\geq 0$ fiber or vector space as fibers, with compact structure group acting by isometry, then $E \times \mathbb{R}^{p}$ admits a complete metric with positive Ricci curvature for all sufficiently large $p$. See $[\mathbf{1 1 0}]$ for an estimate of $p$.

Surgery constructions are very successful in constructing manifolds with positive scalar curvature, see Rothenberg's article in this volume. Sha-Yang $[94,95]$ showed that this is also a useful method for constructing manifolds with positive Ricci curvature in special cases. In particular they showed that if $M^{m+1}$ has a complete metric with Ric $>0$, and $n, m \geq 2$, then $S^{n-1} \times$ $\left(M^{m+1} \backslash \coprod_{i=0}^{k} D_{i}^{m+1}\right) \bigcup_{I d} D^{n} \times \coprod_{i=0}^{k} S_{i}^{m}$, which is diffeomorphic to $\left(S^{n-1} \times M^{m+1}\right) \#\left(\#_{i=1}^{k} S^{n} \times S^{m}\right)$, carries a complete metric with Ric $>0$ for all $k$, showing that the total Betti number of a compact Riemannian $n$-manifold $(n \geq 4)$ with positive Ricci curvature could be arbitrarily large. See also [6], and [111] when the gluing map is not the identity.

Note that a compact homogeneous space admits an invariant metric with positive Ricci curvature if and only if the fundamental group is finite [78, Proposition 3.4]. This is extended greatly by Grove-Ziller [55] showing that any cohomogeneity one manifold $M$ admits a complete invariant metric with nonnegative Ricci curvature and if $M$ is compact then it has positive Ricci curvature if and only if its fundamental group is finite (see also [93]). Therefore, the fundamental group is the only obstruction to a compact manifold admitting a positive Ricci curvature metric when there is enough symmetry. It remains open what the obstructions are to positive Ricci curvature besides the restriction on the fundamental group and those coming from positive scalar curvature (such as the $\hat{A}$-genus).

Of course, another interesting class of examples are given by Einstein manifolds. For these, besides the "bible" on Einstein manifolds [12], one can refer to the survey book [62] for the development after [12], and the recent articles $[\mathbf{1 4}, \mathbf{1 3}]$ for Sasakian Einstein metrics and compact homogenous Einstein manifolds.

\section{References}

[1] U. Abresch and D. Gromoll, On complete manifolds with nonnegative Ricci curvature, J. Amer. Math. Soc., 3(2) (1990), 355-374.

[2] M.T. Anderson, Convergence and rigidity of manifolds under Ricci curvature bounds, Invent. Math., 102(2) (1990), 429-445.

[3] M.T. Anderson, Metrics of positive Ricci curvature with large diameter, Manuscripta Math., 68(4) (1990), 405-415.

[4] M.T. Anderson, On the topology of complete manifolds of nonnegative Ricci curvature, Topology, 29(1) (1990), 41-55.

[5] M.T. Anderson, Short geodesics and gravitational instantons, J. Differential Geom., 31(1) (1990), 265-275.

[6] M.T. Anderson, Hausdorff perturbations of Ricci-flat manifolds and the splitting theorem, Duke Math. J., 68(1) (1992), 67-82. 
[7] M.T. Anderson and J. Cheeger, Diffeomorphism finiteness for manifolds with Ricci curvature and $L^{n / 2}$-norm of curvature bounded, Geom. Funct. Anal., 1(3) (1991), 231-252.

[8] E. Aubry, Finiteness of $\pi_{1}$ and geometric inequalities in almost positive Ricci curvature, preprint.

[9] I. Belegradek and G. Wei, Metrics of positive Ricci curvature on bundles, Int. Math. Res. Not., 57 (2004), 3079-3096.

[10] L. Bérard-Bergery, Certains fibrés à courbure de Ricci positive, C.R. Acad. Sci. Paris Sér. A-B, 286(20) (1978), A929-A931.

[11] L. Bérard-Bergery, Quelques exemples de variétés riemanniennes complètes non compactes à courbure de Ricci positive, C.R. Acad. Sci. Paris Sér. I Math., 302(4) (1986), 159-161.

[12] A.L. Besse, Einstein manifolds, volume 10 of Ergebnisse der Mathematik und ihrer Grenzgebiete (3) [Results in Mathematics and Related Areas (3)], Springer-Verlag, Berlin, 1987.

[13] C. Böhm, M. Wang, and W. Ziller, A variational approach for compact homogeneous Einstein manifolds, Geom. Funct. Anal., 14(4) (2004), 681-733.

[14] C. Boyer, K. Galicki, Sasakian Geometry and Einstein Metrics on Spheres, Perspectives in Riemannian geometry, 47-61, CRM Proc. Lecture Notes, 40, Amer. Math. Soc., Providence, RI, 2006.

[15] D. Burago, Y. Burago, and S. Ivanov, A course in metric geometry, volume 33 of Graduate Studies in Mathematics, American Mathematical Society, Providence, RI, 2001.

[16] P. Buser, A note on the isoperimetric constant, Ann. Sci. École Norm. Sup. (4), 15(2) (1982), 213-230.

[17] E. Calabi, An extension of E. Hopf's maximum principle with an application to Riemannian geometry, Duke Math. J., 25 (1958), 45-56.

[18] I. Chavel, Riemannian Geoemtry: A Modern Introduction, volume 108 of Cambridge Tracts in Mathematics, Cambridge University Press, Cambridge, 1993.

[19] J. Cheeger, Differentiability of Lipschitz functions on metric measure spaces, Geom. Funct. Anal., 9(3) (1999), 428-517.

[20] J. Cheeger, Integral bounds on curvature elliptic estimates and rectifiability of singular sets, Geom. Funct. Anal., 13(1) (2003), 20-72.

[21] J. Cheeger, T.H. Colding, and G. Tian, On the singularities of spaces with bounded Ricci curvature, Geom. Funct. Anal., 12(5) (2002), 873-914.

[22] J. Cheeger, Critical points of distance functions and applications to geometry, in 'Geometric topology: recent developments' (Montecatini Terme, 1990), 1504 of Lecture Notes in Math., 1-38, Springer, Berlin, 1991.

[23] J. Cheeger, Degeneration of Riemannian metrics under Ricci curvature bounds, Lezioni Fermiane [Fermi Lectures], Scuola Normale Superiore, Pisa, 2001.

[24] J. Cheeger, Degeneration of Einstein metrics and metrics with special holonomy, in 'Surveys in differential geometry', VIII (Boston, MA, 2002), Surv. Differ. Geom., VIII, 29-73, Int. Press, Somerville, MA, 2003.

[25] J. Cheeger and T.H. Colding, Lower bounds on Ricci curvature and the almost rigidity of warped products, Ann. of Math. (2), 144(1) (1996), 189-237.

[26] J. Cheeger and T.H. Colding, On the structure of spaces with Ricci curvature bounded below I, J. Differential Geom., 46(3) (1997), 406-480.

[27] J. Cheeger and T.H. Colding, On the structure of spaces with Ricci curvature bounded below II, J. Differential Geom., 54(1) (2000), 13-35.

[28] J. Cheeger and T.H. Colding, On the structure of spaces with Ricci curvature bounded below III, J. Differential Geom., 54(1) (2000), 37-74. 
[29] J. Cheeger and D.G. Ebin, Comparison theorems in Riemannian geometry, North-Holland Publishing Co., Amsterdam, 1975, North-Holland Mathematical Library, 9.

[30] J. Cheeger and D. Gromoll, The splitting theorem for manifolds of nonnegative Ricci curvature, J. Differential Geometry, 6 (1971/72), 119-128.

[31] J. Cheeger and G. Tian, Anti-self-duality of curvature and degeneration of metrics with special holonomy, Comm. Math. Phys., 255(2) (2005), 391-417.

[32] J. Cheeger and G. Tian, Curvature and injectivity radius estimates for Einstein 4-manifolds, J. Amer. Math. Soc., 19(2) (2006), 487-525 (electronic).

[33] J. Cheeger and S.-T. Yau, A lower bound for the heat kernel, Comm. Pure Appl. Math., 34(4) (1981), 465-480.

[34] S.Y. Cheng and S.-T. Yau, Differential equations on Riemannian manifolds and their geometric applications, Comm. Pure Appl. Math., 28(3) (1975), 333-354.

[35] S.Y. Cheng, Eigenvalue comparison theorems and its geometric applications, Math. Z., 143(3) (1975), 289-297.

[36] T.H. Colding, Large manifolds with positive Ricci curvature, Invent. Math., 124(1-3) (1996), 193-214.

[37] T.H. Colding, Ricci curvature and volume convergence, Ann. of Math. (2), 145(3) (1997), 477-501.

[38] M.G. Crandall and P.-L. Lions, Viscosity solutions of Hamilton-Jacobi equations, Trans. Amer. Math. Soc., 277(1) (1983), 1-42.

[39] C.B. Croke, An eigenvalue pinching theorem, Invent. Math., 68(2) (1982), 253-256.

[40] X. Dai, P. Petersen, V, and G. Wei, Integral pinching theorems. Manu. Math., 101 (2000), 143-152.

[41] X. Dai and G. Wei, A heat kernel lower bound for integral Ricci curvature, Michigan Math. Jour., 52 (2004), 61-69.

[42] Y. Ding, Heat kernels and Green's functions on limit spaces, Comm. Anal. Geom., 10(3) (2002), 475-514.

[43] K. Fukaya, Collapsing of Riemannian manifolds and eigenvalues of Laplace operator, Invent. Math., 87(3) (1987), 517-547.

[44] K. Fukaya and T. Yamaguchi, The fundamental groups of almost non-negatively curved manifolds, Ann. of Math. (2), 136(2) (1992), 253-333.

[45] S. Gallot. Inégalités isopérimétriques, courbure de Ricci et invariants géométriques, I, C.R. Acad. Sci. Paris Sér. I Math., 296(7) (1983), 333-336.

[46] S. Gallot, Isoperimetric inequalities based on integral norms of Ricci curvature, Astérisque, 157-158 (1988), 191-216, Colloque Paul Lévy sur les Processus Stochastiques (Palaiseau, 1987).

[47] D. Gilbarg and N.S. Trudinger, Elliptic partial differential equations of second order, Classics in Mathematics. Springer-Verlag, Berlin, 2001, reprint of the 1998 edition.

[48] P.B. Gilkey, J.H. Park, and W. Tuschmann, Invariant metrics of positive Ricci curvature on principal bundles, Math. Z., 227(3) (1998), 455-463.

[49] D. Gromoll, Spaces of nonnegative curvature, in 'Differential geometry: Riemannian geometry' (Los Angeles, CA, 1990), volume 54 of Proc. Sympos. Pure Math., 337-356, Amer. Math. Soc., Providence, RI, 1993.

[50] M. Gromov, Curvature, diameter and Betti numbers, Comment. Math. Helv., 56(2) (1981), 179-195.

[51] M. Gromov, Groups of polynomial growth and expanding maps, Inst. Hautes Études Sci. Publ. Math., 53 (1981), 53-73.

[52] M. Gromov, Metric structures for Riemannian and non-Riemannian spaces, volume 152 of Progress in Mathematics, Birkhäuser Boston Inc., Boston, MA, 1999; Based on the 1981 French original [MR 85e:53051], With appendices by M. Katz, P. Pansu and S. Semmes, Translated from the French by S.M. Bates. 
[53] K. Grove, P. Petersen, V, and J.Y. Wu, Geometric finiteness theorems via controlled topology, Invent. Math., 99(1) (1990), 205-213.

[54] K. Grove and K. Shiohama, A generalized sphere theorem, Ann. Math. (2), 106(2) (1977), 201-211.

[55] K. Grove and W. Ziller, Cohomogeneity one manifolds with positive Ricci curvature, Invent. Math., 149(3) (2002), 619-646.

[56] R.S. Hamilton, Three-manifolds with positive Ricci curvature, J. Differential Geom., $\mathbf{1 7}(\mathbf{2})$ (1982), 255-306.

[57] L. Hörmander, Notions of convexity, volume 127 of Progress in Mathematics, Birkhäuser Boston Inc., Boston, MA, 1994.

[58] H. Ishii, On the equivalence of two notions of weak solutions, viscosity solutions and distribution solutions, Funkcial. Ekvac., 38(1) (1995), 101-120.

[59] V. Kapovitch and B. Wilking, Fundamental groups of manifolds with lower Ricci curvature bounds, in preparation.

[60] B. Kawohl and N. Kutev, Strong maximum principle for semicontinuous viscosity solutions of nonlinear partial differential equations, Arch. Math. (Basel), 70(6) (1998), 470-478.

[61] J. Kollar, Einstein metrics on connected sums of $S^{2} \times S^{3}$, J. Differential Geom., 75 (2) (2007), 259-272.

[62] C. LeBrun and McKenzie Wang, editors, Surveys in differential geometry: essays on Einstein manifolds, Surveys in Differential Geometry, VI, International Press, Boston, MA, 1999; Lectures on geometry and topology, sponsored by Lehigh University's Journal of Differential Geometry.

[63] P. Li, Large time behavior of the heat equation on complete manifolds with nonnegative Ricci curvature, Ann. of Math. (2), 124(1) (1986), 1-21.

[64] P. Li, Lecture notes on geometric analysis, volume 6 of Lecture Notes Series, Seoul National University, Research Institute of Mathematics Global Analysis, Research Center, Seoul, 1993.

[65] A. Lichnerowicz, Géométrie des groupes de transformations, Travaux et Recherches Mathématiques, III, Dunod, Paris, 1958.

[66] P.-L. Lions, Optimal control of diffusion processes and Hamilton-Jacobi-Bellman equations. II. Viscosity solutions and uniqueness, Comm. Partial Differential Equations, 8(11) (1983), 1229-1276.

[67] W. Littman, A strong maximum principle for weakly L-subharmonic functions, J. Math. Mech., 8 (1959), 761-770.

[68] J. Lohkamp, Metrics of negative Ricci curvature, Ann. of Math. (2), 140(3) (1994), 655-683.

[69] J. Lott, Collapsing and the differential form Laplacian: the case of a smooth limit space, Duke Math. J., 114(2) (2002), 267-306.

[70] J. Lott, Some geometric properties of the Bakry-Émery-Ricci tensor, Comment. Math. Helv., 78(4) (2003), 865-883.

[71] J. Lott, Remark about the spectrum of the p-form Laplacian under a collapse with curvature bounded below, Proc. Amer. Math. Soc., 132(3) (2004), 911-918 (electronic).

[72] X. Menguy, Examples of nonpolar limit spaces, Amer. J. Math., 122(5) (2000), 927937.

[73] X. Menguy, Noncollapsing examples with positive Ricci curvature and infinite topological type, Geom. Funct. Anal., 10(3) (2000), 600-627.

[74] X. Menguy, Examples of strictly weakly regular points, Geom. Funct. Anal., 11(1) (2001), 124-131.

[75] J. Milnor, A note on curvature and fundamental group, J. Differential Geometry, 2 (1968), $1-7$. 
[76] S. Myers, Riemannian manifolds with positive mean curvature, Duke Math. J., 8 (1941), 401-404.

[77] P. Nabonnand, Sur les variétés riemanniennes complètes à courbure de Ricci positive, C.R. Acad. Sci. Paris Sér. A-B, 291(10) (1980), A591-A593.

[78] J.C. Nash, Positive Ricci curvature on fibre bundles, J. Differential Geom., 14(2) (1979), 241-254.

[79] M. Obata, Certain conditions for a Riemannian manifold to be isometric with a sphere, J. Math. Soc. Japan, 14 (1962), 333-340.

[80] Y. Otsu, On manifolds of positive Ricci curvature with large diameter, Math. Z., 206(2) (1991), 255-264.

[81] G. Perelman, A.D. Aleksandrov spaces with curvatures bounded below. Part II, preprint.

[82] G. Perelman, Manifolds of positive Ricci curvature with almost maximal volume, J. Amer. Math. Soc., 7(2) (1994), 299-305.

[83] G. Perelman, A complete Riemannian manifold of positive Ricci curvature with Euclidean volume growth and nonunique asymptotic cone, in 'Comparison geometry' (Berkeley, CA, 1993-94), volume 30 of Math. Sci. Res. Inst. Publ., 165-166, Cambridge Univ. Press, Cambridge, 1997.

[84] G. Perelman, Construction of manifolds of positive Ricci curvature with big volume and large Betti numbers, in 'Comparison geometry' (Berkeley, CA, 1993-94), volume 30 of Math. Sci. Res. Inst. Publ., 157-163, Cambridge Univ. Press, Cambridge, 1997.

[85] G. Perelman, The entropy formula for the Ricci flow and its geometric applications, math.DG/0211159.

[86] P. Petersen, Riemannian geometry, volume 171 of Graduate Texts in Mathematics, Springer-Verlag, New York, 1998.

[87] P. Petersen, V, On eigenvalue pinching in positive Ricci curvature, Invent. Math., 138(1) (1999), 1-21.

[88] P. Petersen, V, and C. Sprouse, Integral curvature bounds, distance estimates and applications, J. Differential Geom., 50(2) (1998), 269-298.

[89] P. Petersen, V, and G. Wei, Relative volume comparison with integral curvature bounds, GAFA, 7 (1997), 1031-1045.

[90] P. Petersen, V, and G. Wei, Analysis and geometry on manifolds with integral Ricci curvature bounds, Tran. AMS, 353(2) (2001), 457-478.

[91] W.A. Poor, Some exotic spheres with positive Ricci curvature, Math. Ann., 216(3) (1975), 245-252.

[92] R. Schoen and S.-T. Yau, Complete three-dimensional manifolds with positive Ricci curvature and scalar curvature, in 'Seminar on Differential Geometry', volume 102 of Ann. of Math. Stud., 209-228, Princeton Univ. Press, Princeton, NJ, 1982.

[93] L.J. Schwachhöfer and W. Tuschmann, Metrics of positive Ricci curvature on quotient spaces, Math. Ann., 330(1) (2004), 59-91.

[94] J.-P. Sha and DaGang Yang, Examples of manifolds of positive Ricci curvature, J. Differential Geom., 29(1) (1989), 95-103.

[95] J.-P. Sha and DaGang Yang, Positive Ricci curvature on the connected sums of $S^{n} \times S^{m}$, J. Differential Geom., 33(1) (1991), 127-137.

[96] Z. Shen and C. Sormani, The codimension one homology of a complete manifold with nonnegative Ricci curvature, Amer. J. Math., 123(3) (2001), 515-524.

[97] Z. Shen and C. Sormani, The topology of open manifolds with nonnegative Ricci curvature, math.DG/0606774, preprint.

[98] K. Shiohama, A sphere theorem for manifolds of positive Ricci curvature, Trans. Amer. Math. Soc., 275(2) (1983), 811-819. 
[99] C. Sormani, On loops representing elements of the fundamental group of a complete manifold with nonnegative Ricci curvature, Indiana Univ. Math. J., 50(4) (2001), $1867-1883$.

[100] C. Sormani, The almost rigidity of manifolds with lower bounds on Ricci curvature and minimal volume growth, Comm. Anal. Geom., 8(1) (2000), 159-212.

[101] C. Sormani, Nonnegative Ricci curvature, small linear diameter growth and finite generation of fundamental groups, J. Differential Geom., 54(3) (2000), $547-559$.

[102] C. Sormani and G. Wei, Hausdorff convergence and universal covers, Trans. Amer. Math. Soc., 353(9) (2001), 3585-3602 (electronic).

[103] C. Sormani and G. Wei, Universal covers for Hausdorff limits of noncompact spaces, Trans. Amer. Math. Soc., 356(3) (2004), 1233-1270 (electronic).

[104] C. Sprouse, Integral curvature bounds and bounded diameter, Comm. Anal. Geom., 8(3) (2000), 531-543.

[105] G. Wei, Examples of complete manifolds of positive Ricci curvature with nilpotent isometry groups, Bull. Amer. Math. Soci., 19(1) (1988), 311-313.

[106] G. Wei, On the fundamental groups of manifolds with almost-nonnegative Ricci curvature, Proc. Amer. Math. Soc., 110(1) (1990), 197-199.

[107] G. Wei, Ricci curvature and betti numbers, J. Geom. Anal., 7 (1997), 493-509.

[108] G. Wei, Math. Lecture Notes, 241, http://www.math.ucsb.edu/ wei/241notes.html.

[109] B. Wilking, On fundamental groups of manifolds of nonnegative curvature, Differential Geom. Appl., 13(2) (2000), 129-165.

[110] D. Wraith, Stable bundles with positive Ricci curvature, preprint.

[111] D. Wraith, Surgery on Ricci positive manifolds, J. Reine Angew. Math., 501 (1998), 99-113.

[112] W. Wylie, Noncompact manifolds with nonnegative Ricci curvature, J. Geom. Anal., 16(3) (2006), 535-550.

[113] W. Wylie, On the fundamental group of noncompact manifolds with nonnegative Ricci curvature, Ph.D. thesis at UC Santa Barbara, 2006.

[114] S. Xu, Z. Wang, and F. Yang, On the fundamental group of open manifolds with nonnegative Ricci curvature, Chinese Ann. Math. Ser. B, 24(4) (2003), 469-474.

[115] D. Yang, Convergence of Riemannian manifolds with integral bounds on curvature, I, Ann. Sci. École Norm. Sup. (4), 25(1) (1992), 77-105.

[116] S.-T. Yau, Some function-theoretic properties of complete Riemannian manifold and their applications to geometry, Indiana Univ. Math. J., 25(7) (1976), 659-670.

[117] S.-H. Zhu, A finiteness theorem for Ricci curvature in dimension three, J. Differential Geom., 37(3) (1993), 711-727.

[118] S.-H. Zhu, The comparison geometry of Ricci curvature, in 'Comparison geometry' (Berkeley, CA, 1993-94), volume 30 of Math. Sci. Res. Inst. Publ, 221-262, Cambridge Univ. Press, Cambridge, 1997.

Department of Mathematics, University of California,

SANTA BARbara, CA 93106

E-mail address: wei@math.ucsb.edu 